\title{
On the use of water as a solvent - simple and short one- step synthesis of maleimides
}

\author{
Vladimir Ondruš,* Lubor Fišera*, and Vladimir Bradac \\ Department of Organic Chemistry, Slovak University of Technology, SK-812 37, Bratislava, \\ Slovak Republic \\ E-mail: ondrus@cvt.stuba.sk,fisera@cvt.stuba.sk
}

To Miha Tišler on the occasion of his $75^{\text {th }}$ birthday

(received 30 Jun 01; accepted 23 Oct 01; published on the web 31 Oct 01)

\begin{abstract}
A new route to the synthesis of novel chiral and achiral maleimides is described. A cheap and readily available exo-Diels-Alder adduct of furan and maleic anhydride 1 reacted with amino acids $\mathbf{2 a - d}$ and $\mathbf{4 e - \mathbf { i }}$ in water under classical heating or microwave irradiation with the release of furan to give maleimides 3a-d and 5e-i in good to excellent yields.
\end{abstract}

Keywords: 7-exo-Oxohimic anhydride, chiral maleimides, microwave irradiation

\section{Introduction}

Maleimides are an important class of substrates for biological, pharmacological and chemical applications. In biological applications they are used as chemical probes of protein structure, ${ }^{1}$ as immunoconjugates for cancer therapy, as solid-supported enzymes for synthetic applications, for the productions of antibodies, ${ }^{2}$ in pharmacological applications they are used as analogues of the cyclic tetrapeptide chlamydocin, ${ }^{3}$ photoactivatable fluorescein derivatives, ${ }^{4}$ naltrexone analogues, ${ }^{5}$ or as a new herbicides and pesticides. ${ }^{6} \mathrm{~N}$-(3,5-Dichlorophenyl)pyrrolidine-2,5-dione (Dimetachlon) is being used as a protective and curative fungicide, ${ }^{7}$ and the cycloadduct of $N$ (3,5-dichlorophenyl)maleimide to furan possesses also considerable fungicidal properties. ${ }^{8}$

The maleimide moiety can be used as a versatile platform in synthesis due to Michael accepting ability, dienophilic nature ${ }^{9-11}$ as well as a dipolarophile in 1,3-dipolar cycloadditions. $^{12-14}$

Despite this, there are only a few reports of the synthesis of $N$-substituted chiral maleimides. Most methods involve the reaction of an amine with maleic anhydride, followed by dehydratation of the intermediate maleamic acid, usually promoted by acid. ${ }^{15}$ This method is limited to the use of amines as starting material excluding those, that are unstable under the 
dehydration conditions.

With the goal of developing simple and efficient methods for synthesis of unnatural analogues of natural products from readily available starting material via 1,3-dipolar or DielsAlder cycloadditions, we have focused our attention to simple and effective synthesis of chiral maleimides as dipolarophiles or dienophiles. Probably one of the best possibilities for utilization of this effort offers the 7-exo-oxohimic anhydride $1 .{ }^{16}$ Recently, we have used cheap and readily available exo-Diels-Alder adduct of furan and maleic anhydride $\mathbf{1}$ as a vehicle, which in turn reacted with hydrochlorides of amino acids in the presence of Et $3 \mathrm{~N}$ with the release of furan to give desired novel chiral maleimides. ${ }^{17}$

\section{Results and Discussion}

In this paper we report a simple synthesis of some $N$-substituted maleimides 3a-d and 5e-i derived from chiral amino acids 2a-d, and terminal amino acids 4e-i using either microwave irradiation (Scheme 1) or classical heating (Schemes 2 and 3).

The two most used methods for the synthesis of the required imides were described. Fox and Minard $^{18}$ published the synthesis of $N$-maleonyl-(S)- and $(R)$-valine by agitation of the corresponding amino acid with maleic anhydride at $100{ }^{\circ} \mathrm{C}$ with a yield of about $75 \%$. Rich ${ }^{19}$ prepared the same imides by a two step synthesis from maleic anhydride in the presence of Et $3 \mathrm{~N}$ in toluene with yields 20 - $50 \%$. Recently, Biagini and co-workers ${ }^{20}$ published a simple synthesis of norbornenyl substituted amino acids derived from free amino acids and exo/endohimic anhydride by heating the reaction mixture in DMF or DMSO. Autors reported this method as unsuccesfull in the case of maleic anhydride. ${ }^{20}$ On the basis of this report we have started a search for efficient method for the preparation of $N$-substituted chiral maleimides.

The exo-oxohimic anhydride (1) and $S$-isoleucine (2a) have been chosen and the reaction was performed under conditions described by Bari $^{21}$ for $N$-phthaloyl glycine by using a domestic microwave oven (DMF, power $500 \mathrm{~W}, \mathrm{~N}$-methylpyrrolidine as catalyst). In our case, using exooxohimic anhydride (1) resulted only inseparable mixture of products, no signals for cyclic olefin bond could be detected in ${ }^{1} \mathrm{H}$ and ${ }^{13} \mathrm{C}$ NMR spectra. The same results have been obtained using toluene and DMSO as solvents or by using silica gel as a solid support. ${ }^{22}$

We found that using water as a solvent in the absence of a basic catalyst gave chiral imide 3a in good yield (Method A). Next we tested with success this synthetic route also for other amino acids, and even in the cases of $S$-alanine (2b), S-serine (2c) and $S$-tryptophane (2d) it was successful in shorter reaction time (Scheme 1). In contrast, $S$-glutamic acid and $S$-threonine even after prolongation of reaction time (90 - 120s, 4-6x,) increasing power to $750 \mathrm{~W}$ gave only mixture of uncyclized maleamic acid, maleic acid and starting amino acid. The reaction of 1 with aspartic acid offered only inseparable mixture of maleimides and uncyclized maleamic acid. 


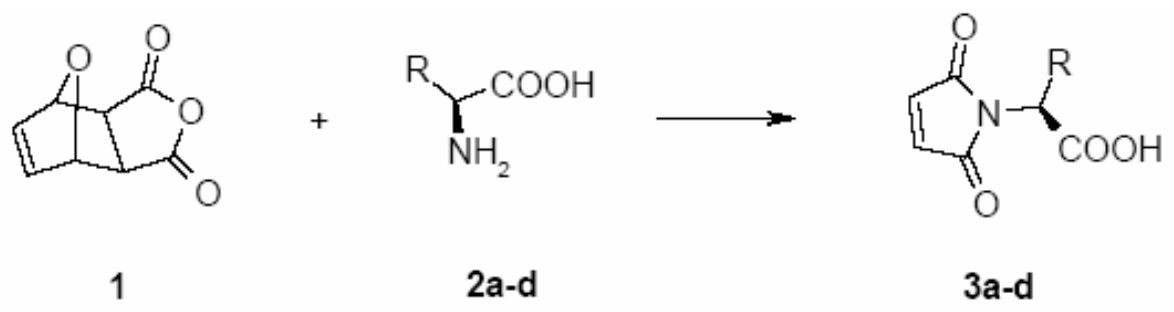

$\begin{array}{ccccc}\text { Set } & \text { R } & \text { Power } & \text { Time } & \text { Yield \% } \\ \text { 3a } & S \text {-Ile } & 500 \mathrm{~W} & 5 \times 60 \mathrm{sec} & 81 \\ \text { 3b } & S \text {-Ala } & 500 \mathrm{~W} & 4 \times 35 \mathrm{sec} & 60 \\ \text { 3c } & S \text {-Ser } & 500 \mathrm{~W} & 5 \times 45 \mathrm{sec} & 92 \\ \text { 3d } & S \text {-Trp } & 500 \mathrm{~W} & 2 \times 60 \mathrm{sec} & 72\end{array}$

\section{Scheme 1}

All of the maleimides prepared in this way were optically active, providing the first indication, that the relatively harsh reaction conditions had not caused complete racemisation of the amino acid hence ${ }^{1} \mathrm{H}$ and ${ }^{13} \mathrm{C}$ NMR spectra of 3a show only one set of signals.

While domestic microwave oven is certainly not a standard equipment, we have tested the above mentioned conditions (water, without catalyst) using conventional heating (Method B). This modified method gave in all cases (Scheme 2) good yields and we were also able to isolate pure $N$-maleonyl glycine $3 \mathbf{e}$ in high yield.

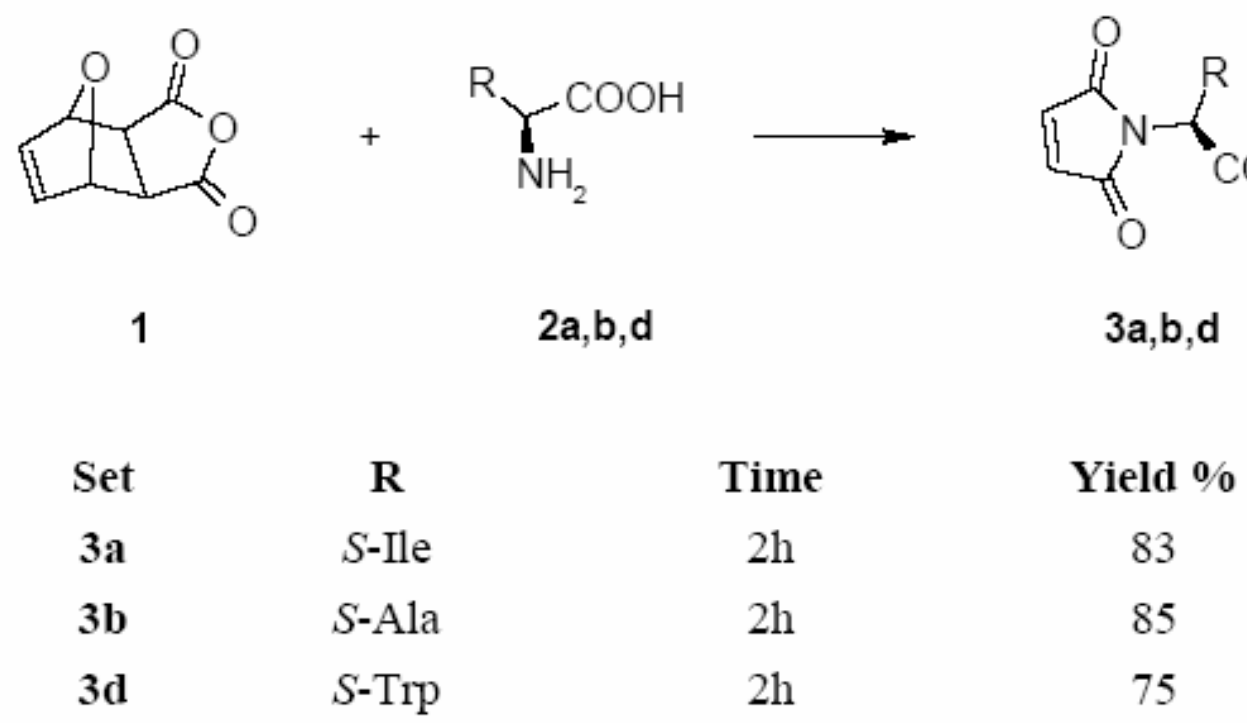

\section{Scheme 2}


This cheap and simple method was further successfully applied to the synthesis of maleimides 5e-i -a potential new types of inhibitors of cyclooxygenase ${ }^{23}$ (Scheme 3 ).

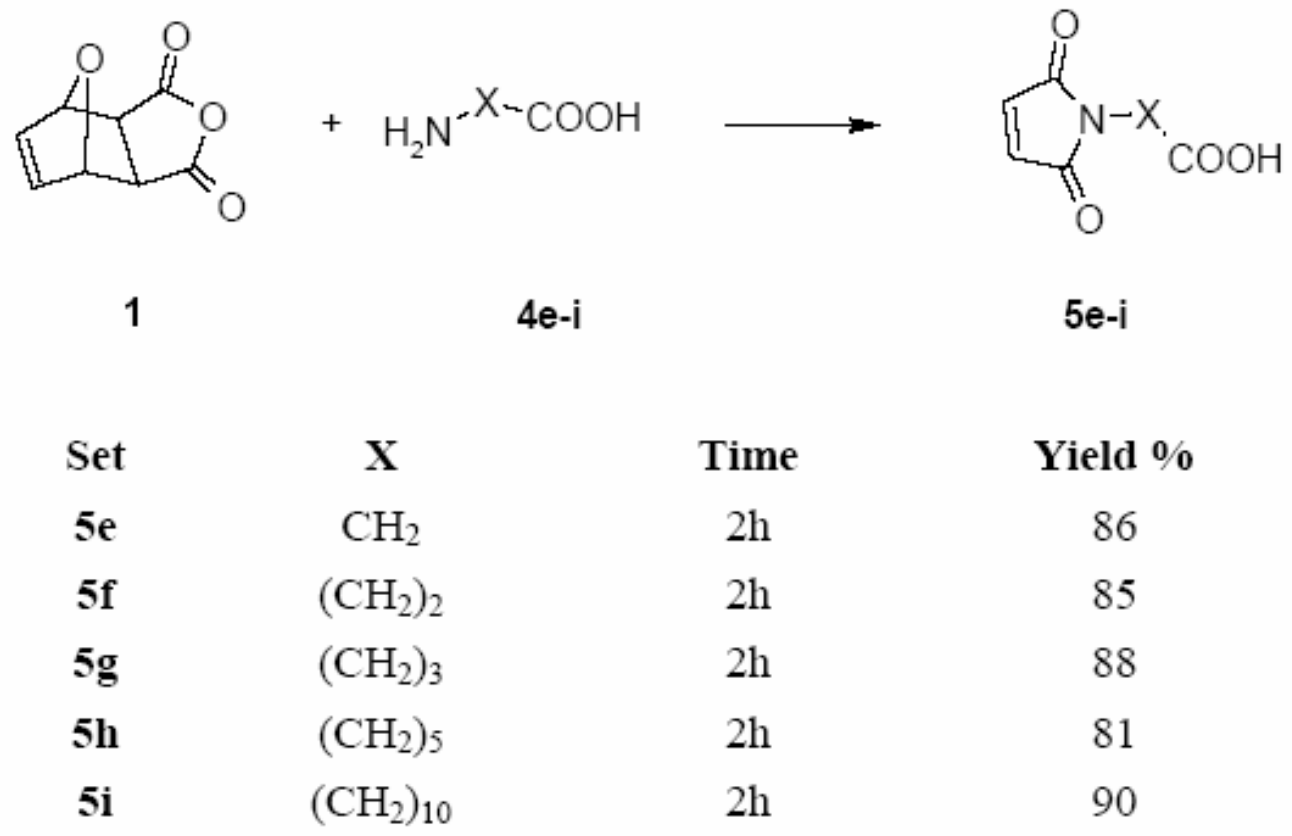

\section{Scheme 3}

\section{Conclusions}

A new route for the synthesis of chiral and achiral maleimides in good to high yields has been developed. This synthetic pathway is very short and simple, using only cheap and available starting materials, reagents, water as a solvent and laboratory equipments. The synthesis of $N$ aminosubstituted maleimides as a possible way for the formation of $N$-maleonyl di- and polypeptides is in progress.

\section{Experimental Section}

General Procedures. ${ }^{1} \mathrm{H}$ NMR spectra were recorded at $300 \mathrm{MHz}$ on a Varian VXR 300 at 293 $\mathrm{K}$ in DMSO. Spectra were internally referenced to HMDS. Peaks are reported downfield of HMDS. Multiplicities are reported as singlet (s), doublet (d), triplet (t), quartet (q), some combination of these, broad (br), or multiplet (m). ${ }^{13} \mathrm{C}$ NMR spectra were recorded at $75.5 \mathrm{MHz}$ on the same spectrometer as ${ }^{1} \mathrm{H}$ NMR at $293 \mathrm{~K}$ in DMSO. Coupling constants are given in $\mathrm{Hz}$ and without sign. Peak assignments were made by DEPT and a * indicates that peak assignments 
may be interchanged. Optical rotations were recorded on POLAR L- $\mu$ P (IBZ-Messtechnik) polarimeter and are reported along with solvent and concentration in $\mathrm{g} / 100 \mathrm{~mL}$. Elemental analyses were obtained on a EA 1108 - Elemental analyzer (Carlo Erba) instrument. Melting points were determined on a Kofler melting point instrument. All yields refer to isolated, analytically pure compounds and have not been optimized. All reactions were performed on domestic microwave oven Whirlpool (Sweden).

Materials. Bicyclic derivative 1 was synthetized by literature procedure. ${ }^{16}$ Commercial amino acids (Avocado, Aldrich) 2a-d and 4e-i were used without purification.

Method A (See Scheme 1). The mixture of bicyclic compound 1 (10.0 mmol) and amino acid 2a-d (10.0 mmol) in $25 \mathrm{~mL}$ water was added in a $100 \mathrm{~mL}$ open Erlenmayer flask and irradiated carefully in domestic microwave as above (Scheme 1). Water was evaporated by azeotropic destillation with toluene in vacuo and the residue was triturated with $30 \mathrm{~mL}$ of acetone until it solidified. Filtration gave analytically pure compounds 3a-d.

Method B (See Schemes 2 and 3). The mixture of bicyclic compound $1(10.0 \mathrm{mmol})$ and amino acids 2a, 2b, 2d and 4e-i (10.0 mmol) in $40 \mathrm{~mL}$ water was stirred and heated under reflux as above (Schemes 2 and 3). Water was evaporated by azeotropic destillation with toluene in vacuo and the residue was triturated with $30 \mathrm{~mL}$ of acetone until it solidified. Filtration gave analytically pure compounds $3 \mathbf{a}, 3 \mathbf{b}, 3 \mathbf{d}$ and $5 \mathbf{e}-\mathbf{i}$.

$N$-Maleonyl-(S)-isoleucine (3a). Method A: Yield $81 \%$. For $\mathrm{C}_{10} \mathrm{H} 13 \mathrm{NO}_{4} \cdot 2 \mathrm{H}_{2} \mathrm{O}$ M.W. 247.25. Calc (\%): C 48.58, H 6.93, N 5.67. Found (\%): C 48.31, H 6.79, N 5.70. $[\alpha]_{\mathrm{D}}{ }^{25}=+13.4$ (c $=2$, $\mathrm{MeOH})$ M.p. $128-9^{\circ} \mathrm{C}$. Method B: Yield $83 \%$. For $\mathrm{C} 10 \mathrm{H}_{13} \mathrm{NO}_{4} \cdot 2 \mathrm{H}_{2} \mathrm{O}$ M.W. 247.25. Calc (\%): C 48.58, H 6.93, N 5.67. Found (\%): C 48.65, H 7.02, N 5.79. $[\alpha]^{25}=+14.3(\mathrm{c}=1.9, \mathrm{MeOH})$ M.p. $127-9{ }^{\circ} \mathrm{C} .{ }^{1} \mathrm{H}$ NMR $\delta 0.89\left(\mathrm{dd}, J=6.9, J=7.5,3 \mathrm{H}, \mathrm{CH}_{2} \mathrm{CH}_{3}\right), 0.92(\mathrm{~d}, J=9.3,3 \mathrm{H}$, $\left.\mathrm{CHCH}_{3}\right), 1.26\left(\mathrm{~m}, 1 \mathrm{H}, \mathrm{CH}_{2}\right), 1.43\left(\mathrm{~m}, 1 \mathrm{H}, \mathrm{CH}_{2}\right), 1.86(\mathrm{~m}, 1 \mathrm{H}, \mathrm{CH}), 3.81(\mathrm{~d}, J=3.7,1 \mathrm{H}, \mathrm{CH}-\mathrm{N})$, $6.08(\mathrm{~s}, 2 \mathrm{H}, \mathrm{CH}=\mathrm{CH}), 8.10(\mathrm{br} \mathrm{s}, 1 \mathrm{H}, \mathrm{COOH}) ;{ }^{13} \mathrm{C}$ NMR $\delta 11.2,14.0\left(2 \mathrm{x} \mathrm{q}, 2 \times \mathrm{CH}_{3}\right), 24.7(\mathrm{t}$, $\left.\mathrm{CH}_{2}\right), 35.4(\mathrm{~d}, \mathrm{CH}), 55.8(\mathrm{~d}, \mathrm{CH}-\mathrm{N}), 134.7(2 \times \mathrm{d}, \mathrm{C}=\mathrm{C}), 166.9(2 \times \mathrm{s}, 2 \times \mathrm{C}=\mathrm{O}) *$, 169.9 (s, $\mathrm{COOH})$.

$N$-Maleonyl-(S)-alanine (3b). Method A: Yield $60 \%$. For $\mathrm{C}_{7} \mathrm{H}_{7} \mathrm{NO}_{4} \cdot 2 \mathrm{H}_{2} \mathrm{O}$ M.W. 205.17. Calc (\%): C 40.98, H 5.40, N 6.83. Found (\%): C 40.84, H 5.27, N 6.70. $[\alpha]_{\mathrm{D}}^{25}=+4.7(\mathrm{c}=2$, $\mathrm{MeOH})$ M.p. $155-7^{\circ} \mathrm{C}$. Method B: Yield $86 \%$. For $\mathrm{C}_{7} \mathrm{H}_{7} \mathrm{NO}_{4} \cdot 2 \mathrm{H}_{2} \mathrm{O}$ M.W. 205.17. Calc (\%): C 40.98, H 5.40, N 6.83. Found (\%): C 40.54, H 5.44, N 6.79. $[\alpha]_{\mathrm{D}} 25=+4.6(\mathrm{c}=1.9, \mathrm{MeOH})$ M.p. $154-7{ }^{\circ} \mathrm{C} .{ }^{1} \mathrm{H}$ NMR $\delta 1.36\left(\mathrm{~d}, \mathrm{~J}=7.2,3 \mathrm{H}, \mathrm{CH}_{3}\right), 3.92(\mathrm{q}, 1 \mathrm{H}, \mathrm{CH}), 6.08(\mathrm{~s}, 2 \mathrm{H}, \mathrm{CH}=\mathrm{CH})$, 8.20 (br s, $1 \mathrm{H}, \mathrm{COOH}) ;{ }^{13} \mathrm{C}$ NMR $\delta 15.9\left(\mathrm{q}, \mathrm{CH}_{3}\right), 48.0(\mathrm{~d}, \mathrm{CH}), 135.2(2 \mathrm{x} \mathrm{d}, \mathrm{C}=\mathrm{C}), 167.3$ (s, $\mathrm{COOH}), 171.7$ ( 2 x s, 2 x C=O).

$N$-Maleonyl-(S)-serine (3c). Method A: Yield $92 \%$. For $\mathrm{C}_{7} \mathrm{H}_{7} \mathrm{NO}_{5} .2 \mathrm{H}_{2} \mathrm{O}$ M.W. 221.15. Calc (\%): C 38.02, H 5.01, N 6.93. Found (\%): C 38.23, H 4.94, N 6.38. $[\alpha]_{\mathrm{D}}^{25}=+3.0(\mathrm{c}=2$, $\mathrm{MeOH})$ M.p. $124-5{ }^{\circ} \mathrm{C} .{ }^{1} \mathrm{H}$ NMR $\delta 3.78\left(\mathrm{ddd}, J=3.9, J=3.9, J=6.0,2 \mathrm{H}, \mathrm{CH}_{2}\right), 3.91(\mathrm{dd}, 1 \mathrm{H}$, $\mathrm{CH}-\mathrm{N}), 6.08(\mathrm{~s}, 2 \mathrm{H}, \mathrm{CH}=\mathrm{CH}), 8.10(\mathrm{br} \mathrm{s}, 1 \mathrm{H}, \mathrm{COOH}) ;{ }^{13} \mathrm{C}$ NMR $\delta 54.6\left(\mathrm{t}, \mathrm{CH}_{2}\right), 59.7(\mathrm{~d}, \mathrm{CH}-$ 
N), $135.6(2 \times$ d, C=C), $167.5(\mathrm{~s}, \mathrm{COOH}), 169.7(2 \times \mathrm{s}, 2 \times \mathrm{C}=\mathrm{O})$.

N-Maleonyl-(S)-tryptophane (3d). Method A: Yield $72 \%$. For $\mathrm{C}_{15} \mathrm{H}_{12} \mathrm{~N}_{2} \mathrm{O}_{4} .2 \mathrm{H}_{2} \mathrm{O}$ M.W.320.30 Calc (\%): C 56.30, H 5.03, N 8.75. Found (\%): C 56.38, H 4.98, N 8.75. [ $\alpha]_{\mathrm{D}}{ }^{25}=-5.8(\mathrm{c}=1.8$, $\mathrm{MeOH})$ M.p. $158-162{ }^{\circ} \mathrm{C}$. Method B: Yield $75 \%$. For $\mathrm{C}_{15} \mathrm{H}_{12} \mathrm{~N}_{2} \mathrm{O}_{4} \cdot 2 \mathrm{H}_{2} \mathrm{O}$ M.W.320.30 Calc (\%): C 56.30, H 5.03, N 8.75. Found (\%): C 56.52, H 4.87, N 8.90. $[\alpha]_{\mathrm{D}}{ }^{25}=-5.5(\mathrm{c}=2.0, \mathrm{MeOH})$ M.p. $162-4{ }^{\circ} \mathrm{C} .{ }^{1} \mathrm{H}$ NMR $\delta 3.26\left(\mathrm{dd}, J=5.0, J=5.9,2 \mathrm{H}, \mathrm{CH}_{2}\right), 4.12(\mathrm{dd}, 1 \mathrm{H}, \mathrm{CH}-\mathrm{N}), 6.07$ (s, 2H, $\mathrm{CH}=\mathrm{CH}), 7.01\left(\mathrm{ddd}, J=7.9, J=7.8, J=0.4,1 \mathrm{H}, \mathrm{H}_{\text {arom }}\right), 7.10\left(\mathrm{ddd}, 1 \mathrm{H}, \mathrm{H}_{\text {arom. }}\right), 7.22(\mathrm{~d}, J=2.3$, $\left.1 \mathrm{H}, \mathrm{H}_{\text {pyrol }}\right), 7.38\left(\mathrm{~d}, 1 \mathrm{H}, \mathrm{H}_{\text {arom }}\right), 7.56\left(\mathrm{~d}, 1 \mathrm{H}, \mathrm{H}_{\text {arom }}\right) ;{ }^{13} \mathrm{C} \mathrm{NMR} \delta 26.4\left(\mathrm{t}, \mathrm{CH}_{2}\right), 52.9(\mathrm{~d}, \mathrm{CH}-\mathrm{N})$, 106.9 (s, $\left.\mathrm{C}_{\text {arom}}\right), 111.7,118.4,118.8,119.6,125.0$ (d, $\left.\mathrm{C}_{\text {arom }}\right), 127.14$ (s, $\left.\mathrm{C}_{\text {arom}}\right), 135.6$ (2 x d, $\mathrm{C}=\mathrm{C}), 136.4$ (s, $\left.\mathrm{C}_{\text {arom}}\right), 167.4$ (s, $\left.\mathrm{COOH}\right), 171.1(2 \times \mathrm{s}, 2$ x C=O).

$N$-Maleonyl glycine (5e). Method B: Yield $86 \%$. For $\mathrm{C}_{6} \mathrm{H}_{5} \mathrm{NO}_{4} \cdot 2 \mathrm{H}_{2} \mathrm{O}$ M.W. 191.11. Calc (\%): $\mathrm{C} 37.71, \mathrm{H} 4.74, \mathrm{~N} 7.32$. Found (\%): C 37.57, H 4.63, N 7.09. M.p. $118-121{ }^{\circ} \mathrm{C} .{ }^{1} \mathrm{H}$ NMR $\delta 3.75$ $\left(\mathrm{s}, 2 \mathrm{H}, \mathrm{CH}_{2}\right), 6.04(\mathrm{~s}, 2 \mathrm{H}, \mathrm{CH}=\mathrm{CH}), 8.23($ br s, $1 \mathrm{H}, \mathrm{COOH}) ;{ }^{13} \mathrm{C} \mathrm{NMR} \delta 51.2\left(\mathrm{t}, \mathrm{CH}_{2}\right), 134.8(2$ $\mathrm{x} \mathrm{d}, \mathrm{C}=\mathrm{C}), 167.3$ (s, $\mathrm{COOH}), 174.2(2 \times \mathrm{s}, 2$ x C=O).

$N$-Maleonyl-3-aminopropanoic acid (5f). Method B: Yield $85 \%$. For $\mathrm{C}_{7} \mathrm{H}_{7} \mathrm{NO}_{4} \cdot 2 \mathrm{H}_{2} \mathrm{O}$ M.W. 205.17. Calc (\%): C 40.98, H 5.40, N 6.83. Found (\%): C 40.94, H 5.51, N 6.96. M.p. 117$120{ }^{\circ} \mathrm{C} .{ }^{1} \mathrm{H}$ NMR $\delta 2.57\left(\mathrm{dd}, J=6.6, J=7.2,2 \mathrm{H}, \mathrm{CH}_{2}-\mathrm{COOH}\right)^{*}, 3.00\left(\mathrm{dd}, 2 \mathrm{H}, \mathrm{CH}_{2}-\mathrm{N}\right) *, 6.07(\mathrm{~s}$, $2 \mathrm{H}, \mathrm{CH}=\mathrm{CH}) ;{ }^{13} \mathrm{C}$ NMR $\delta 31.7\left(\mathrm{t}, \mathrm{CH}_{2}\right), 35.0(\mathrm{t}, \mathrm{CH} 2-\mathrm{N}), 136.2(2 \mathrm{x} \mathrm{d}, \mathrm{C}=\mathrm{C}), 167.7(\mathrm{~s}, \mathrm{COOH})$, $172.2(2 \times \mathrm{s}, 2 \times \mathrm{C}=\mathrm{O})$.

$N$-Maleonyl-4-aminobutanoic acid (5g). Method B: Yield $88 \%$. For $\mathrm{C}_{8} \mathrm{H}_{9} \mathrm{NO}_{4} \cdot 2 \mathrm{H}_{2} \mathrm{O}$ M.W. 219.19. Calc (\%): C 43.84, H 5.98, N 6.39. Found (\%): C 43.72, H 5.92, N 6.41. M.p. 106-8 ${ }^{\circ} \mathrm{C}$. ${ }^{1} \mathrm{H}$ NMR $\delta 1.76$ (dddd, $\left.J=7.3, J=7.3, J=7.5, J=7.6,2 \mathrm{H}, \mathrm{CH}_{2}-\mathrm{CH} 2-\mathrm{CH} 2\right), 2.33$ (dd, 2H, CH2$\mathrm{N})^{*}, 2.83(\mathrm{dd}, 2 \mathrm{H}, \mathrm{CH} 2-\mathrm{COOH})^{*}, 6.07(\mathrm{~s}, 2 \mathrm{H}, \mathrm{CH}=\mathrm{CH}), 8.00$ (br s, $\left.1 \mathrm{H}, \mathrm{COOH}\right) ;{ }^{13} \mathrm{C} \mathrm{NMR} \delta$ $22.6(\mathrm{t}, \mathrm{CH} 2-\mathrm{CH} 2-\mathrm{CH} 2), 30.6(\mathrm{t}, \mathrm{CH} 2-\mathrm{N})^{*}, 38.5(\mathrm{t}, \mathrm{CH} 2-\mathrm{COOH})^{*}, 136.2(2 \mathrm{x} \mathrm{d}, \mathrm{C}=\mathrm{C}), 167.6$ (s, $\mathrm{COOH}), 173.9(2 \times \mathrm{s}, 2 \times \mathrm{C}=\mathrm{O})$.

$N$-Maleonyl-6-aminohexanoic acid (5h). Method B: Yield 81 \%. For $\mathrm{C}_{10} \mathrm{H}_{13} \mathrm{NO}_{4} .2 \mathrm{H}_{2} \mathrm{O}$ M.W. 247.25. Calc (\%): C 48.58, H 6.93, N 5.67. Found (\%): C 48.32, H 6.92, N 5.44. M.p. 107$110{ }^{\circ} \mathrm{C} .{ }^{1} \mathrm{H}$ NMR $\delta 1.32(\mathrm{~m}, 2 \mathrm{H}, \mathrm{CH} 2), 1.51(\mathrm{~m}, 4 \mathrm{H}, 2 \mathrm{xCH} 2), 2.21(\mathrm{dd}, J=7.1, J=7.2,2 \mathrm{H}$, $\mathrm{CH} 2-\mathrm{N}) *, 2.78(\mathrm{dd}, J=7.3, J=7.9,2 \mathrm{H}, \mathrm{CH} 2-\mathrm{COOH})^{*}, 6.04(\mathrm{~s}, 2 \mathrm{H}, \mathrm{CH}=\mathrm{CH}) ;{ }^{13} \mathrm{C}$ NMR $\delta 24.0$, 25.4, 26.8, 33.4, 38.8 (t, CH2), 136.2 ( 2 x d, C=C), 167.4 (s, COOH), 174.4 ( 2 x s, 2 x C=O).

$N$-Maleonyl-11-aminoundecanoic acid (5i). Method B: Yield $90 \%$. For C15H23NO4. 2 H2O M.W. 317.38. Calc (\%): C 56.77, H 8.57, N 4.33. Found (\%): C 56.83, H 8.83, N 4.33. M.p. 113$5{ }^{\circ} \mathrm{C} .{ }^{1} \mathrm{H}$ NMR $\delta 1.24$ (br s, $\left.12 \mathrm{H}, 6 x \mathrm{CH} 2\right), 1.48$ (m, 4H, 2xCH2), 2.19 (dd, $J=7.2, J=7.5,2 \mathrm{H}$, $\mathrm{CH} 2-\mathrm{N})^{*}, 2.78(\mathrm{dd}, J=7.5, J=7.8,2 \mathrm{H}, \mathrm{CH} 2 \mathrm{COOH})^{*}, 6.05(\mathrm{~s}, 2 \mathrm{H}, \mathrm{CH}=\mathrm{CH}) ;{ }^{13} \mathrm{C} \mathrm{NMR} \delta 24.6$, 25.8, 27.0, 28.5, 28.6, 28.8, 33.7, 38.9, 39.2 (t, CH2), 136.2 (2 x d, C=C), $167.4(\mathrm{~s}, \mathrm{COOH})$, 174.6 ( 2 x s, $2 \times \mathrm{C}=\mathrm{O})$. 


\section{Acknowledgements}

The authors are grateful to the Slovak Grant Agency (No. 1/7314/20) and Volkswagenstiftung Hannover for financial support.

\section{References}

1. Corrie, J. E. T. J. Chem. Soc., Perkin Trans. 1 1994, 2975.

2. Janda, K. D.; Ashley, J. A.; Jones, T. M.; McLeod, D. A.; Schloeder, D. M.; Weinhouse, M. I. J. Am. Chem. Soc. 1990, 112, 8886.

3. Rich, D. H.; Jasensky, R. D., Mueller, G. C.; Anderson, K. E. J. Med. Chem. 1981, $24,567$.

4. Corrie, J. E. T.; Trentham, D. R. J. Chem. Soc., Perkin Trans. 1 1995, 1993.

5. Sayre, M.; Larson, D. L.; Takemori, E.; Portoghese, P. S.; J. Med. Chem. 1984, $27,325$.

6. Matocsy, G.; Nadasi, M.; Adriska, V. In Pesticide Chemistry, Akademiai Kiadó: Budapest 1988.

7. Fujinami, A.; Ozaki, T.; Nodera, K.; Tanaka, K.; Agric. Biol. Chem. 1972, 36, 318.

8. Tottori, N.; Ueda, M.; Kirino, O.; Oba, S.; Fujinami, A.; Kato, T.; Ozaki, T.; Japan Kokai 124 225; Chem. Abstr. 1975, 82, 150501.

9. Baldwin, S. P.; Greenspan, P.; Alaimo, C.; McPhail, A. T. Tetrahedron Lett. 1991, 32, 5877.

10. Philp, D.; Robertson, A. J. Chem. Soc., Chem. Commun. 1998, 879.

11. Bravo, P. A.; Pozo Carero, M. C.; Román Galán, E.; Serano Blázquez, J. A. Heterocycles 2000, 53, 81.

12. Grigg, R.; Surendrakumar, S.; Thiampatanagul, S.; Vipond, D.J. J. Chem. Soc., Perkin Trans. 1 1988, 2693.

13. (a) Konopíková, M.; Fišera, L.; Prónayová, N. Collect. Czech. Chem. Commun. 1991, 57, 1521. (b) Blanáriková, I.; Dugovič, B.; Fišera, L.; Hametner, C. ARKIVOC 2001, 2, 1091.

14. Philp, D.; Booth, C. A. Tetrahedron Lett. 1998, 39, 6987.

15. Searle, N. E.; U. S. Pat. 2444 536, 1948, Chem. Abstr. 1948, 42, 7340.

16. Tochtermann, W.; Bruhu, S.; Wolff, C. Tetrahedron Lett. 1994, 35, 1165.

17. Ondruš, V.; Fišera, L. Molecules 1997, 2, 49.

18. Fox, S. W.; Minard, F. N. J. Am. Chem. Soc. 1952, 74, 2085.

19. Rich, D. H.; Gesellchen, P. D.; Tong, A.; Cheung, A.; Buckner, C. K. J. Med. Chem. 1975, 18, 1004.

20. Biagini, S. C. G.; Bush, S. M.; Gibson, V. C.; Mazzariol, L.; North, M.; Teasdale, W. G.; Wiliams, C. M.; Zagotto, G.; Zamuner, D. Tetrahedron 1995, 51, 7247.

21. Bari, S. S.; Bose, A. K.; Chaudhary, A. G.; Manhas, M. S.; Raju, V. S.; Robb, E. W. J. Chem. Educ. 1992, 69, 938.

22. Borah, H. N.; Boruah, R. C., Sandhu, J. S. J. Chem. Res.(S) 1998, 5, 272. 
23. Marnet, L. J.; Kalkutkar, A. S.; WO 9515163; Chem. Abstr. 1993, 123, 160 835n. 